Earlier Use of High Doses of poMTX and scMTX in 2nd Time Period Daching RA Treatment Targets
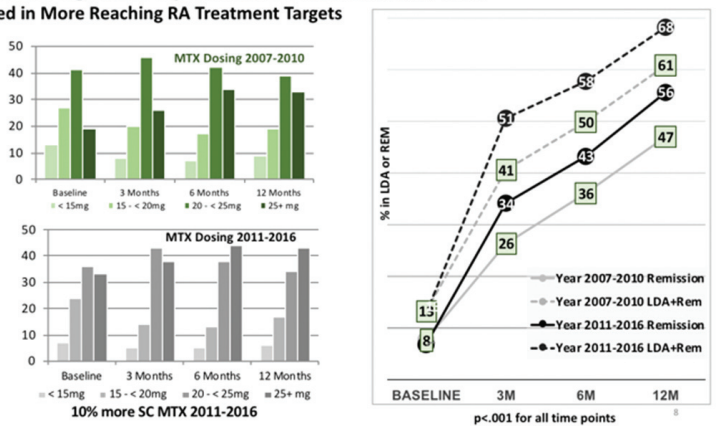

Abstract SAT0080 - Figure 1. Earlier Use of High Doses of poMTX and ScMTX in 2nd Time Period Resulted in More Reaching RA Treatment Targets

Conclusions: This 10 year Canadian prospective study of classifiable/probable RA patients, who were assessed for 1 year suggests that earlier, more intensified treatment promoted in practice recommendations were implemented and resulted in lower disease activity with a greater proportion of patients reaching targets of LDA and/or REM, although $25 \%-30 \%$ of patients still did not achieve LDA or REM by $12 \mathrm{M}$.

Acknowledgements: Sponsors: Amgen \& Pfizer-Founding sponsors 2007+; UCB, AbbVie 2011+; Medexus 2013+; Eli Lilly, Sanofi- Genzyme 2016+; Merck 2017+; BMS 2011-2014, Hoffmann-LaRoche, Janssen 2011-2016

Disclosure of Interest: None declared

DOI: 10.1136/annrheumdis-2018-eular.7268

\section{SAT0081 CHANGES IN INCIDENCE OF SHOULDER, ELBOW, WRIST AND FINGER REPLACEMENT SURGERY AMONG RHEUMATOID ARTHRITIS PATIENTS FOLLOWING THE INTRODUCTION OF BIOLOGICAL DMARDS: AN INTERRUPTED TIME SERIES ANALYSIS USING DANISH HEALTH CARE REGISTERS}

R.L. Cordtz ${ }^{1}$, S. Hawley ${ }^{2}$, D. Prieto-Alhambra ${ }^{2}$, P. Højgaardd ${ }^{1,3}$, K. Zobbe $^{1}$, A. Odgaard ${ }^{4}$, S. Overgaard ${ }^{5}$, L.E. Kristensen ${ }^{3}$, L. Dreyer ${ }^{1,3,6} .{ }^{1} C^{2}$ Center for Rheumatology and Spine Diseases, Rigshospitalet-Gentofte, Copenhagen, Denmark; ${ }^{2}$ Musculoeskeletal Pharmaco- and Device Epidemiology, Centre for Statistics in Medicine, Nuffield Department of Orthopaedics, Rheumatology and Musculoskeletal Sciences, University of Oxford, Oxford, UK; ${ }^{3}$ The Parker Institute, Bispebjerg and Frederiksberg Hospital, Frederiksberg; ${ }^{4}$ Department of Orthopaedic Surgery, Copenhagen University Hospital Herlev- Gentofte, Copenhagen; ${ }^{5}$ Department of Orthopaedic Surgery and Traumatology, University Hospital Odense, Odense; ${ }^{6}$ Department of Rheumatology, Aalborg University Hospital, Aalborg, Denmark

Background: We have previously shown that the incidence rate of total knee replacements started to decrease among rheumatoid arthritis (RA) patients following the introduction of biological (b) DMARDs, but less is known on the impact of bDMARDs on the need for joint replacements (JR) of the upper limbs.

Objectives: To investigate the association between bDMARD introduction for the treatment of patients with RA on the trends of upper limb JR among newly diagnosed RA patients compared with a matched general population cohort (GPC) in Denmark.

Methods: Nationwide register-based interrupted time-series analysis using the Danish National Patient Register and Civil Registration System. Study populations: incident RA patients diagnosed at a rheumatology or general internal medicine clinic/department from 1996-2010. Intervention: introduction of bDMARDs in Denmark in 2002. Comparison: Each RA patient was matched on age, sex and municipality with up to 10 non-RA individuals (GPC). Outcome: Composite outcome of first shoulder, elbow, wrist, or finger replacement surgery (JR). Statistical analyses: 5 year age- and sex-standardised incidence rates of JR calculated for incident RA patients diagnosed biannually compared with GPCs. Outcome trends in the pre-bDMARD era (1996-2001) were compared with those in the bDMARD era (2003-2015) with a 1 year lag period in 2002.

Results: From 1996 to 2010, 26458 incident RA patients were identified and compared with 257,505 GPCs (Table). The JR incidence rate was stable among RA patients in 1996-2001, but started to decrease from 2003 and onwards. Among GPCs, the incidence rate increased throughout the study period.

Stepwise backward elimination to produce most parsimonious model: $p$-entry $<0.05$ and $p$ exit $>0.2$.

* $\triangle$ per year based on biannual data. Abbreviations: pyrs, person years

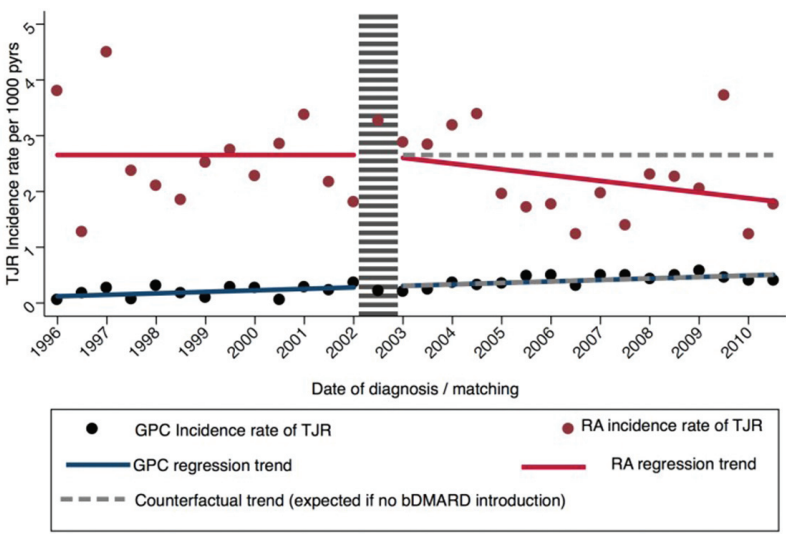

Conclusions: Following the introduction of bDMARDs, the incidence rate of upper limb JR started to decrease among RA patients, whereas the incidence rate steadily increased from 1996-2015 among matched GPCs. The baseline incidence rate was 7 -fold higher among RA patients than GPCs, but the absolute need for upper limb JR was low in both groups.

Disclosure of Interest: R. Cordtz: None declared, S. Hawley: None declared, D. Prieto-Alhambra Grant/research support from: Amgen, Servier, and UCB, Consultant for: UCB, Speakers bureau: Amgen, P. Højgaard Speakers bureau: UCB and Celgene, K. Zobbe: None declared, A. Odgaard: None declared, S. Overgaard: None declared, L. E. Kristensen Speakers bureau: Pfizer, AbbVie, Amgen, UCB BMS, Biogen, MSD, Novartis, Eli Lilly and Company, and Janssen Pharmaceuticals, L. Dreyer Speakers bureau: UCB and MSD DOI: 10.1136/annrheumdis-2018-eular.2328

\section{SAT0082 OSTEOPONTINE AND OSTEOPROTEGRINE AS MARKERS OF ALTERED PRECLINICAL BONE METABOLISM IN RHEUMATOID FIRST DEGREE RELATIVES}

E. Soliman ${ }^{1}$, M. Zehairy ${ }^{2}$, A. Aly ${ }^{2}$, K. Mattarawy ${ }^{3}$, A. alhadidy ${ }^{2} .{ }^{1}$ Internal Medicine, Rheumatology and Clinical Immunology, ${ }^{2}$ Faculty of Medicine; ${ }^{3}$ medical research, Alexandria, Egypt

Background: First degree relatives (FDR) of RA are known to have increased risk of developing the disease. The detection of altered bone metabolism in FDR could be a predictor of the preclinical phase of the disease.

Objectives: To study osteopontine (OPN) and osteoprotegrine (OPG) in FDR of RA patients as markers of altered bone metabolism in relation to clinical manifestations, inflammatory and RA seromarkers.

Abstract SAT0081 - Table 1. Changes in 5-year incidence rate of upper limb joint replacements (JR) in incident rheumatoid arthritis (RA) patients following introduction of biological DMARDs in 2002 compared with secular trends in a matched general population cohort (GPC).

\begin{tabular}{|c|c|c|c|c|c|c|c|c|c|c|}
\hline Cohort & $N$ & $\begin{array}{l}\text { Mean age at start } \\
\text { of follow-up }\end{array}$ & $\begin{array}{l}\text { Females, } \\
\text { n (\%) }\end{array}$ & n JR & PYRS & $\begin{array}{l}\text { Baseline incidence } \\
\text { rate/1000 pyrs }\end{array}$ & $\begin{array}{c}\triangle \text { per } \\
\text { year* }^{\star} \\
1996- \\
2001\end{array}$ & $\begin{array}{l}\triangle \text { in } \\
\text { level } \\
2003\end{array}$ & $\begin{array}{c}\triangle \text { per } \\
\text { year }^{\star} \\
2003- \\
2015\end{array}$ & $\begin{array}{c}\text { Absolute/relative } \triangle \text { at midpoint in bDMARD era (mid- } \\
\text { 2006) compared with counterfactual value }\end{array}$ \\
\hline$\overline{R A}$ & 26458 & $\begin{array}{r}58.9 \\
\text { years }\end{array}$ & $\begin{array}{l}18691 \\
(71 \%)\end{array}$ & 295 & 123814 & $\begin{array}{c}2.65 \\
(2.27-3.04)\end{array}$ & - & - & $\begin{array}{c}-0.10 \\
(-0.21- \\
+0.01)\end{array}$ & $-0.44(-0.49$ to -0.39$) /-17 \%$ \\
\hline GPC & 257505 & $\begin{array}{r}58.4 \\
\text { years }\end{array}$ & $\begin{array}{c}182192 \\
(71 \%)\end{array}$ & 377 & $1,152,052$ & $\begin{array}{c}0.11 \\
(0.04-0.17)\end{array}$ & $\begin{array}{c}0.03 \\
(0.02- \\
0.03)\end{array}$ & - & $\begin{array}{c}0.03 \\
(0.02- \\
0.03)\end{array}$ & No change \\
\hline
\end{tabular}


Methods: 55 persons were included, divided into 20 RA patients, 25 FDR of RA patients (without evidence of arthritis) and 10 healthy matched controls. Clinical evaluation, with emphasis on joint symptoms and signs was done for all, in addition to measurement of ESR, CRP, RF, anti-CCP, serum OPN and serum OPG. Results: Mean ESR was significantly higher in RA $(64.15 \pm 34.29)$ than in FDR $(15.6 \pm 11.04, p \leq 0.001)$ and controls $(6.0 \pm 2.05, p \leq 0.001)$ and significantly higher in FDR than controls $(p=0.001)$. Mean CRP was significantly higher in RA (26.38 $\pm 29.14)$ than FDR $(5.99 \pm 5.08, p \leq 0.001)$ and controls $(2.02 \pm 0.53, p \leq 0.001)$ and sig nificantly higher in FDRs than in controls $(p=0.011)$. Mean RF and anti-CCP were statistically higher in RA than in FDR and controls. Mean anti-CCP was higher in FDR than in controls but without reaching statistical significance while there was no difference regarding mean RF between FDR and controls. OPN was higher in RA (3.66 \pm 4.20$)$ than in FDR $(1.97 \pm 1.04)$ and controls $(2.81 \pm 1.31)$ without statistical significance $(p=0.102)$. While OPG was significantly higher in RA (143.89 $\pm 365.47)$ than in both FDR $(22.23 \pm 65.73, p=0.009)$ and controls $(6.20 \pm 12.43$, $\mathrm{p}=0.003$ ). Mean serum OPN in RA was higher in RF and CCP positive (3.77 \pm 4.43 and $4.13 \pm 3.48$ respectively) than RF and CCP negative $(2.65 \pm 0.35$ and $3.58 \pm 2.58$ respectively) but without reaching statistical difference. Mean serum OPG in RA was higher in RF and CCP positive (153.15 \pm 384.64 and 161.78 \pm 394.67 respectively) than RF and CCP negative $(60.50 \pm 85.56$ and 42.47 \pm 68.09 respectively) but without reaching statistical difference. $8 / 25$ (32\%) FDR had arthralgia while 17/25 (68\%) FDR were asymptomatic. FDRs with arthralgia had significantly higher ESR $(27.88 \pm 11.22)$ and CRP $(10.36 \pm 5.21)$ than asymptomatic FDR $(9.82 \pm 4.13, \mathrm{p}=0.003)$ and $(3.93 \pm 3.58, \mathrm{p}=0.003)$ respectively. OPG was higher in FDR than in controls and higher in those with arthralgia (51.55 $\pm 114.68)$ than those without $(8.44 \pm 9.67)$ but without reaching statistical difference $(p=0.314)$. Similarly, serum OPN was higher in FDR with arthralgia $(2.09 \pm 1.19)$ than asymptomatic $(1.70 \pm 0.55)$ but also without significant difference $(p=0.620)$. Furthermore, mean RF and anti-CCP were higher in FDR with arthralgia but didn't reach significant difference.

Conclusions: OPN and OPG are markers of altered bone metabolism in RA. Their elevation in FDR than controls denotes a state of altered bone metabolism. Moreover, FDR with arthralgia experience higher levels of OPN, OPG, ESR, $\mathrm{CRP}, \mathrm{RF}$, and anti-CCP than asymptomatic FDR. These findings reflect an ongoing disturbed bone metabolism and inflammation in FDR which could precede the clinical disease phase. Thus, OPN and OPG could serve as markers of altered preclinical bone metabolism in rheumatoid FDR. Results need to be confirmed on larger numbers of FDR.

Disclosure of Interest: None declared

DOI: 10.1136/annrheumdis-2018-eular.2198

\section{SAT0083 THE GENETIC AND CLINICAL PREDICTION MODELS FOR EFFICACY AND HEPATOTOXICITY OF METHOTREXATE IN PATIENTS WITH RHEUMATOID ARTHRITIS}

A. Onishi ${ }^{1}$, M. Nishida ${ }^{2}$, M. Takahashi ${ }^{3}$, Y. Yoshida $^{4}$, M. Kobayashi ${ }^{4}$, S. Kamitsuji ${ }^{5}$, M. Kawate ${ }^{4}$, K. Nishimura ${ }^{6}$, K. Misaki ${ }^{7}$, Y. Nobuhara ${ }^{8}$, S. Hatachi ${ }^{9}$, T. Nakazawa ${ }^{8}$, G. Tsuji ${ }^{2}$, S. Kumagai ${ }^{2,3}$. ${ }^{1}$ Department of Rheumatology and Clinical Immunology, Kobe University Graduate School of Medicine; ${ }^{2}$ Center for Rheumatic diseases, Shinko Hospital; ${ }^{3}$ Shinko Institute for Medical Research; ${ }^{4}$ Sysmex Corporation, Kobe; ${ }^{5}$ StaGen Co. Ltd., Tokyo; ${ }^{6}$ Department of Endocrinology and Rheumatology, Kurashiki Central Hospital, Okayama; ${ }^{7}$ Department of Rheumatology, Kita-Harima Medical Center, Hyogo; ${ }^{8}$ Department of Rheumatology, Osaka saiseikai nakatsu hospital; ${ }^{9}$ Clinical Immunology and Rheumatology, Kitano Hospital, Osaka, Japan

Background: The efficacy and toxicity of methotrexate (MTX) depend on individual patients with rheumatoid arthritis (RA) and are difficult to predict before treatment although MTX is the anchor drug and achieving the treatment target earlier is desirable to prevent progression of structural and functional damage. Our previous studies revealed high predictive accuracy of single nucleotide polymorphisms (SNPs) to predict the efficacy and toxicity of MTX, suggesting the influence of genetic variations in enzymes associated with MTX metabolism and folate metabolic pathway ${ }^{1,2}$. However, higher accuracy and replicability is demanded for clinical application.

Objectives: To develop combined genetic and clinical models to predict the efficacy and hepatotoxicity of MTX.

Methods: Patients with RA under the treatment of MTX according to Japanese guideline for the management of RA with MTX were enrolled. To predict the efficacy and hepatotoxicity, 1971 polymorphisms of 246 enzymes/transporters potentially relevant to pharmacokinetics and pharmacodynamics of MTX were measured by the DMET microarray (Affymetrix Inc.) and direct-sequencing method and clinical variables at baseline were collected. As for efficacy, the EULAR-CRP response criteria was chosen to classify patients with RA as responders (good response) and non-responders (moderate or no response). Hepatotoxicity was defined as either serum AST or ALT levels higher than 1.5 times the upper limit of the normal range. Among SNPs and clinical variables with significant association with outcomes using univariate analyses, stepwise model selection was conducted by Akaike information criterion in logistic regression model and receiver operating characteristic (ROC) analyses were performed. Bootstrapping ( $n=100,000)$ was used to assess the robustness of the results.

Results: A total of 166 patients with RA was included. The median age was 61.5 years with $81.3 \%$ of women. For efficacy, genetic prediction model using 7 SNPs showed area under the curve of ROC (AUC) was 0.822 with sensitivity of $74.3 \%$ and specificity of $76.8 \%$, while combined clinical and genetic model indicated AUC was 0.844 with sensitivity of $81.5 \%$ and specificity of $76.9 \%$. By incorporating clinical variables into the genetic model, overall category-free net reclassification improvement $(\mathrm{NRI})$ was $0.700(\mathrm{p}<0.0001)$ and overall integrated discrimination improvement (IDI) was $0.089(\mathrm{p}=0.0005)$. For hepatotoxicity, genetic prediction model using 7 SNPs showed AUC was 0.783 with sensitivity of $70.0 \%$ and specif icity of $80.0 \%$, while combined clinical and genetic model indicated AUC was 0.906 with sensitivity of $85.1 \%$ and specificity of $87.8 \%$. Overall category-free NRI was $1.122(p<0.0001)$ and overall IDI was $0.279(p<0.0001)$.
Efficacy

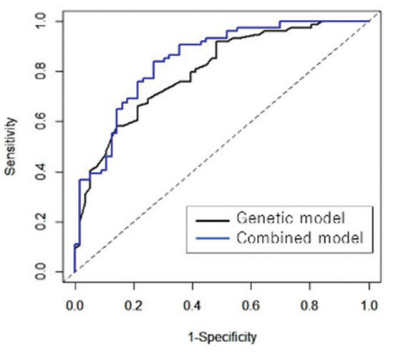

Hepatotoxicity

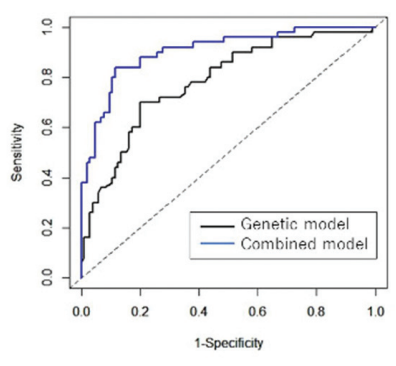

Conclusions: Genetic and clinical models showed higher predictive accuracy for both efficacy and hepatotoxicity of MTX. These models should be validated with a larger scale of prospective study.

REFERENCES:

[1] Kumagai S. Ann Rhem Dis. 2015;74(suppl2):263.

[2] Kumagai S. Ann Rhem Dis. 2014;73(suppl2):119.

Acknowledgements: None.

Disclosure of Interest: None declared

DOI: 10.1136/annrheumdis-2018-eular.5239

\section{SAT0084 ANTI-CARBAMYLATED PROTEIN ANTIBODIES AS POTENTIAL BIOMARKERS OF DISEASE ACTIVITY IN EARLY ARTHRITIS PATIENTS}

A. Gonzalez ${ }^{1}$, C. Regueiro ${ }^{1}$, A.M. Ortiz ${ }^{2}$, L. Nuño ${ }^{3}$, D. Peiteado ${ }^{3}$, A. Villalba $^{3}$, D. Pascual-Salcedo ${ }^{3}$, A. Martinez-Feito ${ }^{3}$, A. Balsa $^{3}$, I. Gonzalez-Alvaro ${ }^{2}{ }^{1}$ Instituto Investigacion Sanitaria- H. Clinico Universitario de Santiago, Santiago de Compostela; ${ }^{2}$ Hospital Universitario La Princesa. IIS-IP; ${ }^{3}$ Instituto de Investigacion del Hospital de La Paz (IDIPAZ), Madrid, Spain

Background: It has become increasingly clear that appropriate initial management of rheumatoid arthritis (RA) increases the chances of success and improves long-term prognosis. Therefore, rheumatologists need prognostic biomarkers to select patients requiring aggressive management. It is possible that anti-carbamylated protein antibodies (anti-CarPA) may serve as such biomarkers because they are associated with erosions and their progression, and with mortality in some studies. Recently, the possibility that they are also associated with disease activity in early arthritis (EA) has been examined by several studies with discordant results.

Objectives: We aimed to explore the relationship between variation in disease activity and anti-CarPA in EA patients.

Methods: EA patients from two prospective clinics, Hospital Universitario La Paz $(\mathrm{n}=492)$ and Hospital Universitario La Princesa $(\mathrm{n}=501)$, were included. DAS28 was available at baseline and at months 6 (M6), 12 (M12) and 24 (M24) of followup. Anti-CarPA were determined in baseline serum samples by ELISA using in vitro carbamylated fetal calf serum. Student t test and main effects general linear regression were used for analysis.

Results: The $27.4 \%$ of EA patients that were positive for anti-CarPA showed higher DAS28 at baseline than the negative patients ( 4.93 vs $\left.4.31, \mathrm{p}=1.6 \times 10-{ }^{8}\right)$. The difference persisted at all visits during follow-up (3.60 vs 3.19 at M6; 3.47 vs 3.09 at M12; and 3.31 vs 2.79 at M24; all with $p \leq 0.001)$. These differences were independent of patient sex and age, smoking, time since symptoms onset, the specific EA clinic and the year of onset. In addition, they persisted after accounting for the presence of RF or ACPA at baseline $\left(p=1.3 \times 10-^{5}\right.$, and $p=5.7 \times 10^{5}{ }^{5}$, respectively) and at later visits ( $p<0.05$ for all analyses). Analysis of the relation 